\title{
Optic nerve sheath diameter with intracranial pressure monitoring: a non-invasive method to follow children with craniosynostosis
}

\author{
Diámetro de la vaina del nervio óptico con monitorización de la presión intracraneal: un \\ método no invasivo para el seguimiento de niños con craneosinostosis
}

\author{
Ahmet Besir', Ali Akdogan ${ }^{1 *}$, and Ali Riza Guvercin² \\ ${ }^{1}$ Department of Anesthesiology and Critical Care; ${ }^{2}$ Department of Neurosurgery. Faculty of Medicine, Karadeniz Technical University, Faculty of \\ Medicine, Trabzon, Turkey
}

\begin{abstract}
Craniosynostosis (CS) is associated with increased intracranial pressure (ICP) and this elevation is of vital importance in children. Bedside optic nerve sheath diameter (ONSD) on ocular ultrasonography has begun to be increasingly used in recently. A patient who was diagnosed with CS, standard anesthesia monitoring and anesthesia were performed. Before and after the surgery, ONSD measurement was performed to follow the changes in ICP. ONSD measurement can be used as an effective, non-invasive, repeated, and easy-to-apply method to monitor the changes in the ICP in pediatric patients with CS who are planned to undergo craniectomy.
\end{abstract}

Key words: Craniosynostosis. Craniofacial surgery. Optic nerve sheat diameter. Intracranial pressure monitoring.

\section{Resumen}

La craneosinostosis (SC) se asocia con un aumento de la presión intracraneal (PIC) y esta elevación es de vital importancia en los niños. El diámetro de la vaina del nervio óptico al lado de la cama (ONSD) en la ecografía ocular ha comenzado a usarse cada vez más recientemente. A un paciente al que se le diagnosticó SC, se le realizó seguimiento anestésico estándar y anestesia. Antes y después de la cirugía, se realizó la medición ONSD para seguir los cambios en la PIC. La medición ONSD se puede utilizar como un método eficaz, no invasivo, repetido y fácil de aplicar para monitorear los cambios en la PIC en pacientes pediátricos con SC que se planea someter a craniectomía.

Palabras clave: Craneosinostosis. Cirugía craneofacial. Diámetro de la vaina del nervio óptico. Monitorización de la presión intracraneal.

\section{Correspondence:}

*Ali AKDOGAN 


\section{Introduction}

Craniosynostosis (CS) is a condition associated with increased intracranial pressure (ICP) due to the premature fusion of cranial sutures leading to restriction in the growth of the brain'. The prevalence of increased ICP in children with syndromic CS due to multiple suture fusion varies $40-70 \%{ }^{2}$. It can be due to hydrocephalus, airway obstruction, craniocerebral disproportion, or abnormalities in the venous drainage of the brain ${ }^{2}$. Depending on all these reasons, complications ranging from mild symptoms to death may occur due to increased ICP. In CS, cranial vault expansion surgery is performed to provide the most appropriate conditions to maximize the optimal condition of the brain or to prevent high ICP.

Increased intracranial pressure (ICP) can lead to permanent neurological sequelae. In patients with CS with high ICP, it is crusial to accelerate ICP-lowering measures and to maintain adequate cerebral perfusion $^{3}$. In the literature, studies on the measurement of ONSD accompanied by USG, which is a noninvasive method in the detection of ICP increase, started in the early 90 s. Especially in the last decade, it has been used frequently in adult and pediatric intensive care units as well as in the operating room during the intraoperative period.

Monitoring of ICP is widely used for the treatment and evaluation of patients with CS, who have elevated $\mathrm{ICP}^{4}$. Although invasive methods are the gold standard for monitoring ICP increase, serious complications such as infection and bleeding may occur in these procedures ${ }^{5,6}$. Although there are less invasive methods for ICP monitoring in pediatric and neonatal patients, because of their disadvantages, bedside optic nerve sheath diameter (ONSD) on ocular ultrasonography (USG) has begun to be increasingly used by pediatricians and intensive care specialists in recent years as it is a noninvasive method ${ }^{6}$.

Here, we presented the importance of ultrasound-guided ONSD measurements, a noninvasive method, in the diagnosis of ICP and ICP monitoring before and immediately after craniectomy in a child with $\mathrm{CS}$.

\section{Case Report}

An eight-month-old male patient with a weight of $10 \mathrm{~kg}$, who was diagnosed with CS, was decided to be operated by the neurosurgery department. In the preoperative evaluation of the patient, no additional problems were observed in his medical history, laboratory findings, and seizure history. Following a threehour of fasting, the patient was transferred to the operating room and standard anesthesia monitoring (pulse oximetry, electrocardiography, non-invasive blood pressure, end-tidal carbon dioxide, temperature, and bispectral index) (Spacelabs Medical, USA) was performed. Then, oropharyngeal airways, laryngeal masks, and other essential equipment at varying sizes were prepared for the probability of difficult intubation. After achieving mask ventilation, anesthesia was induced with IV atropine $(0.01 \mu \mathrm{g} / \mathrm{kg}-1)$, midazolam $(0.5 \mathrm{mg})$, fentanyl $(1 \mu \mathrm{g} / \mathrm{kg}-1)$, and propofol $(3 \mathrm{mg} / \mathrm{kg}-1)$. Endotracheal intubation was successfully performed using a $3.5-\mathrm{mm}$ uncuffed endotracheal tube. Confirmation of endotracheal tube placement was performed with capnography and lung auscultation. Before and after the surgery, USG-guided ONSD measurement was performed to follow the changes in ICP. Volume-controlled ventilation was performed, with a tidal volume and respiratory frequency maintaining normocapnia (EtCO2 32-35 mmHg). No problem arose in the follow-up of hemodynamics and oxygenation. Furthermore, gas concentration of inhaled anesthetics was closely monitored throughout the surgery to monitor possible changes.

Following intubation, anesthesia was maintained with remifentanil and propofol administered in intermittent bolus injection of 50:50 oxygen/air mixture titrated to bispectral index (BIS) value 50-60. No muscle relaxant was used throughout the surgical procedure. Fluid management was performed by the administration of balanced electrolyte solution based on the calculation of the perioperative fluid eficits and basal fluid requirement of the patient.

The ONSD value decreased from the preoperative value of $6.6 \mathrm{~mm}$ to the postoperative value of $5.8 \mathrm{~mm}$ (Fig. 1). During the surgical procedure, an appropriate surgical drape and skin sterilization were performed with the head flexed at 30 degrees while the patient was in the supine position and the skin was elevated in the form of a biparietal flap. Subgaleal dissection was performed without peeling the periosteum to keep the blood loss at the minimum level. The sagittal suture was removed by performing a 5 -cm strip craniectomy. Barrel osteotomies were performed on the temporoparietal bones on both sides to expand the biparietal narrowing and suturectomy was performed on both coronal and lambdoid sutures. Then, frontal and occipital bones were reshaped by performing barrel osteotomies (Fig. 2). Surgical procedure lasted for 


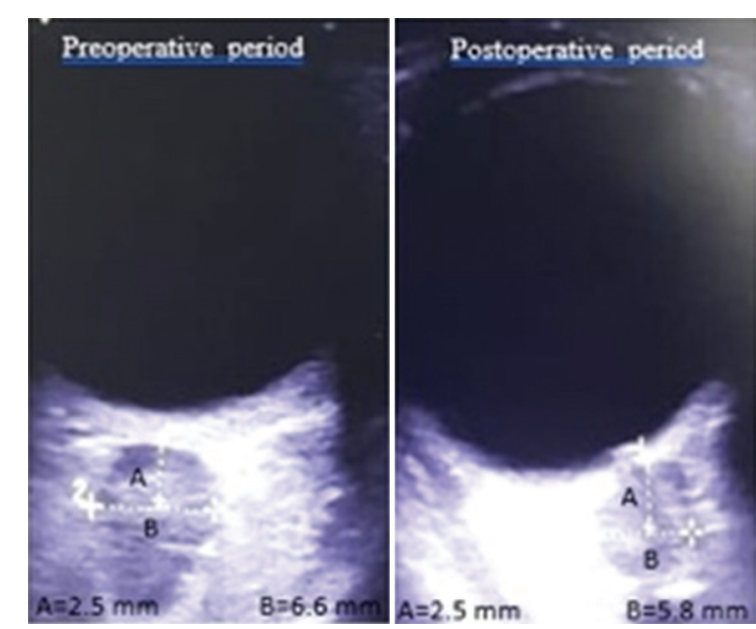

Figure 1. Preoperative and postoperative ONSD measurements.

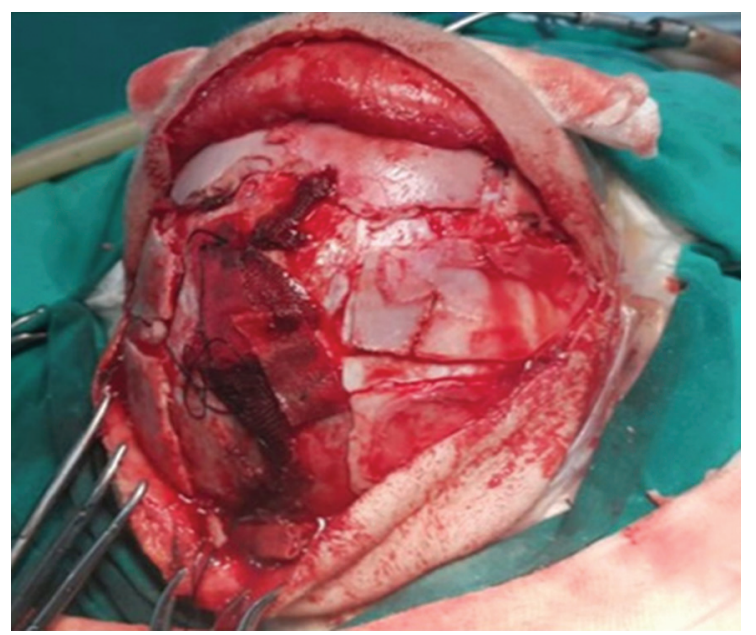

Figure 2. Operational show of the patient with craniosynostosis.

125 minutes and extubation was achieved uneventfully when the patient was full-awake. The patient was, then, transferred to the neonatal intensive care unit.

\section{Discussion}

Children with CS are at great risk for the development of increased ICP. The common clinical symptoms associated with increased ICP include headache, sleep disturbance, irritability, developmental delay, and decreased intellectual abilities ${ }^{7,8}$. Many underlying pathomechanisms have been reported related to the cause of increased ICP due to CS. Craniocerebral disproportion, hydrocephalus, abnormal venous drainage, and secondary extracranial factors are thought to affect ICP in these patients ${ }^{9,10}$.
Complications that may result in brain damage or even death as a result of cerebral ischemia and herniation due to uncontrolled ICP increase may occur. Monitoring of ICP is a clinically objective method that guides the physician in the evaluation and treatment of craniosynostosis. Furthermore, identifying patients with high ICP can help determine whether early intervention is required to prevent cognitive and neurological dysfunction.

In cases with CS, invasive ICP monitoring is widely used to confirm the suspicion of intracranial hypertension before performing corrective surgery ${ }^{3}$. Direct methods of ICP monitoring are the gold standard and are measured via epidural, subdural, and intraparenchymal intracranial catheter, as well as ventricular cannulation $^{11}$. Conventional non-invasive methods used to determine ICP (e.g. Funduscopic examination, neuropsychiatric evaluation, bony changes on $\mathrm{CT}$ ) often yield clear results ${ }^{12}$. In recent years, a new telemetric ICP monitoring system has been also used as a non-invasive and easy-to-apply method ${ }^{13}$. Although cranial magnetic resonance imaging or computed tomography is useful in the diagnosis of ICP in children, exposure to radiation for tomography imaging and the requirement for sedation in the pediatric age group are the disadvantages of these radiological examinations $^{14}$. The fundoscopic examination is also very challenging for newborns since it requires pupil dilation and is impractical15,16.

Monitoring the changes is in ICP, which is one of the comorbidities that worsen the patient's condition before and after decompressive surgery, is of great importance. This will be guiding for the success of the surgery, as well as the patient's follow-up and treatment process. In monitoring these vital functions, there has been always a need for a minimally invasive and fast method of obtaining ICP measurements.

Bedside sonographic ophthalmic ultrasound measurement of ONSD is an easy, inexpensive, non-invasive, and reproducible technique that is commonly used in adults and children to detect ICP. One of the major advantages of this technique is that it does not expose the patient to any radiation ${ }^{7,17-19}$. The subarachnoid space is continuous with the optic nerve perineural space ${ }^{20}$. Changes in ONSD reflect the changes in ICP since there is a direct communication between the subarachnoid space and the optic nerve ${ }^{21}$. Increased ICP and increased CSF between the optic nerve and optic nerve sheath results in an enlarged ONSD since the cavity between the dura mater, optic nerve, and the optic nerve sheath is surrounded by the brain and cerebrospinal fluid ${ }^{22}$. 
In the literature, there are many studies and meta-analyses investigating the normal value of ONSD in adult and pediatric age groups. Since the present case is included in the pediatric age group, the normal upper limit for ONSD has been reported to be $4.0 \mathrm{~mm}$ in children under one year of age and $4.5 \mathrm{~mm}$ in patients over one year of age ${ }^{23}$.

The present case report aimed to determine the level of preoperatively increased ICP in the early postoperative period and to reveal its clinical implications with serial ONSD measurements in an infant with CS undergoing craniectomy.

In the present case, measurements were performed preoperatively and postoperatively by placing a 7.5$\mathrm{MHz}$ linear USG probe on the closed upper eyelid of the patient without excessive pressure after applying a thin layer of sterile gel at room temperature. Then, the ONSD was measured $2.5 \mathrm{~mm}$ behind the optic globe, from the transverse and sagittal planes. All measurements were performed twice, and the mean values were recorded.

Measurements showed that the postcraniectomy ONSD value was lower than the precraniectomy ONSD value (5.8 $\mathrm{mm}$ and $6.6 \mathrm{~mm}$, respectively) (Fig. 1). This decrease in ONSD after craniectomy showed that ICP decreased and the surgery performed was effective.

\section{Conclusion}

In conclusion, USG-guided ONSD measurement can be used as an effective, non-invasive, repeated, and easy-to-apply method with no radiation hazard to monitor the changes in the ICP in pediatric patients with CS who are planned to undergo craniectomy.

\section{Conflicts of interest}

All authors have no conflicts of interest regarding this study.

\section{Ethical disclosures}

Protection of human and animal subjects. The authors declare that no experiments were performed on humans or animals for this study.

Confidentiality of data. The authors declare that they have followed the protocols of their work center on the publication of patient data.

Right to privacy and informed consent. The authors have obtained the written informed consent of the patients or subjects mentioned in the article. The corresponding author is in possession of this document.

\section{References}

1. Thompson DN, Harkness W, Jones B, U Andar, R Hayward. Subdural intracranial pressure monitoring in craniosynostosis: its role in surgical management. Childs Nerv Syst 1995;11:269-275.

2. Thomas $\mathrm{K}$, Hughes $\mathrm{C}$, Johnson $\mathrm{D}$, Das $\mathrm{S}$. Anesthesia for surgery related to craniosynostosis: a review. Part 1. Paediatr Anaesth. 2012;22:1033-1041.

3. Joseph B, Haider AA, Pandit V, Tang A, Kulvatunyou N, O'Keeffe T, et al. Changing paradigms in the management of 2184 patients with traumatic brain injury. Ann Surg. 2015 Sep;262(3):440-8; discussion 446-8. doi: 10.1097/SLA.0000000000001418. PMID: 26258312.

4. Tamburrini G, Caldarelli M, Massimi L, Santini P, Di Rocco C. Intracranial pressure monitoring in children with single suture and complex craniosynostosis: a review. Childs Nerv Syst 2005;21:913-921

5. Arabi Y, Memish ZA, Balkhy HH, Francis C, Ferayan A, Al Shimemeri A, et al. Ventriculostomy-associated infections: incidence and risk factors. Am J Infect Control 2005;33:137-143.

6. Dasic D, Hanna SJ, Bojanic S, Kerr R S C. External ventricular drain infection: the effect of a strict protocol on infection rates and a review of the literature. Br J Neurosurg 2006;20:296-300.

7. Bortcosh W, Shaahinfar A, Sojar S, Klig J E. New directions in point-of care ultra-sound at the crossroads of paediatric emergency and critical care. Curr. Opin. Pediatr. 2018;30:350-358.

8. David LR, Velotta E, Weaver RG, Wilson J A, Argenta L C. Clinical findings precede objective diagnostic testing in the identification of increased ICP in syndromic craniosynostosis. J Craniofac Surg 2002;13:676-680.

9. Gonsalez S, Hayward R, Jones B, Lane R. Upper airway obstruction and raised intracranial pressure in children with craniosynostosis. Eur Respir J 1997;10:367-375.

10. Taylor WJ, Hayward RD, Lasjaunias $P$, Britto J A, Thompson D N Jones $B$ M, et al. Enigma of raised intracranial pressure in patients with complex craniosynostosis: the role of abnormal intracranial venous drainage. J Neurosurg 2001:94:377-385.

11. Wiegand $C$, Richards $P$. Measurement of intracranial pressure in children: a critical review of current methods. Dev Med Child Neurol 2007;49:935-941.

12. Judy B F, Swanson J W, Yang W, Storm PB, Bartlett SP, Taylor JA et al. Intraoperative intracranial pressure monitoring in the pediatric craniosynostosis population. J Neurosurg Pediatr. 2018 1;22:475-480.

13. Beez T, O'Kane R, Piper I, Koppel D, Sangra M. Telemetric Intracranial Pressure Monitoring in Syndromic Craniosynostosis. J Craniofac Surg. 2016;27:1032-1034.

14. Miglioretti D L, Johnson E, Williams A, Greenlee RT, Weinmann S, Solberg $\mathrm{LI}$, et al. The use of computed tomography in pediatrics and the associated radiation exposure and estimated cancer risk. JAMA Pediatr. 2013;167:700-707.

15. Dhaliwal C A, Wright E, Mclntosh N, K Dhaliwal, B W Fleck. Pain in neonates during screening for retinopathy of prematurity using binocular indirect ophthalmoscopy and wide-field digital retinal imaging: a randomized comparison. Arch. Dis. Child. Fetal Neonatal Ed. 2010;95:146-148.

16. Mukherjee A N, Watts $P$, Al-Madfai H, Manoj B, Roberts D. Impact of retinopathy of prematurity screening examination on cardiorespiratory indices: a comparison of indirect oph-thalmoscopy and retcam imaging. Ophthalmology 2006:113:1547-1552.

17. Sinnott J R, Mohebbi M R, Koboldt T. Papilledema: point-of-care ultrasound di-agnosis in the emergency department. Clin Pract Cases Emerg Med 2018;2:125-127.

18. Geeraerts T, Merceron S, Benhamou D, Vigué B, Duranteau J. Non-invasive assessment of in-tracranial pressure using ocular sonography in neurocritical care patients. Intensive Care Med. 2008;34:2062-2067.

19. Dubost C, Le Gouez A, Zetlaoui P J, D Benhamou, F J Mercier, T Geeraerts. Increase in optic nerve sheath diameter induced by epidural blood patch: a preliminary report. Br. J. Anaesth. 2011;107:627-630.

20. Lüdemann W, Berens von Rautenfeld D, Samii M, Brinker T. Ultrastructure of the cere-brospinalfluid outflow along the optic nerve into the lymphatic system. Childs Nerv. Syst. 2005;21:96-103.

21. Shirodkar C G, Rao S M, Mutkule D P, Harde Y R, Venkategowda P M, Mahesh M U. Optic nerve sheath diameter as a marker for evaluation and prognostication of intracranial pressure in Indian pa-tients: an observational study. Indian J Crit Care Med 2014;18:728-734.

22. Helmke $\mathrm{K}$ and Hansen $\mathrm{HC}$. Fundamentals of transorbital sonographic evaluation of optic nerve sheath expansion under intracranial hypertension II. Pediatr Radiol 1996;26):706-710.

23. Gravendal J, Rosendahl K. Cerebral biometry at birth and 4 and 8 months of age. Aprospective study using US. Pediatr. Radiol. 2010;40:1651-1656. 